\title{
Y.I. PETRUKhin
}

\section{Correspondence Analysis for First Degree Entailment}

\author{
Petrukhin Yaroslav Igorevich \\ Department of Logic, Faculty of Philosophy, Lomonosov Moscow State University. \\ Lomonosovsky prospekt, 27-4, GSP-1, Moscow, 119991, Russian Federation. \\ e-mail: yaroslav.petrukhin@mail.ru \\ In this paper natural deduction systems for four-valued logic FDE (first degree \\ entailment) and its extensions are constructed. At that B. Kooi and A. Tamminga's \\ method of correspondence analysis is used. All possible four-valued unary $(\star)$ and binary \\ ( $\circ$ ) propositional connectives which could be added to FDE are considered. Then FDE \\ is extended by Boolean negation $(\sim)$ and every entry (line) of truth tables for $\star$ and \\ $\circ$ is characterized by inference scheme. By adding all inference schemes characterizing \\ truth tables for $\star$ and $\circ$ as rules of inference to the natural deduction for FDE, natural \\ deduction for extension of FDE is obtained. In addition, applying of correspondence \\ analysis gives axiomatizations of implicative extensions of $\mathbf{F D E}$ including $\mathbf{B N}_{4}$ and \\ some extensions by classical implications.
}

Keywords: correspondence analysis, natural deduction, first degree entailment, BelnapDunn logic, four-valued logic, implicative extensions, classical implication

\section{Introduction}

A history of the logic FDE dates back to N.D. Belnap's abstract [5] and A.R. Anderson and N.D. Belnap's paper [1]. They investigate a system of first degree (tautological) entailment which inferences avoid paradoxes of classical entailment and contain connectives $\neg, \wedge$, and $\vee$. An implication is occured in a formula only once: as the main connective. In other words, all first degree formulas are of the form $A \rightarrow B$, where $A$ and $B$ do not contain $\rightarrow$. Since the definitions of $\rightarrow$ and $\models$ are equivalent, $\rightarrow$ is replaced by $\models$ in many papers on this subject (including this one). Moreover, Anderson and Belnap proved that $\mathbf{F D E}$ is a first degree fragment of relevant logic $\mathbf{E}$, i.e. $A \rightarrow B$ (where $A$ and $B$ don't contain $\rightarrow$ ) is provable in $\mathbf{E}$ iff $A \rightarrow B$ is a first degree (tautological) entailment.

There are various semantics for FDE, but in this paper only two of them will be need: N.D. Belnap's semantics [3, 4] and J.M. Dunn's one [8]. They will be discussed in the next section.

The first formalisation of FDE was introduced in [1]. Since then, various studies of proof systems for FDE have been carried out. For this 
paper G. Priest's monography [15] is of particular importance: natural deduction system built in it is actively used here. It should be noted that there are also investigations devoted to extensions of FDE. (Some of them are considered in the section 6.)

This paper is a kind of continuation and generalization of these studies: it is an attempt to explore natural deductions systems axiomatizing all possible truth-table expansions of $\mathbf{F D E}^{1}$. Solving this problem, I use the technique of correspondence analysis, first applied by B. Kooi and A. Tamminga [13] for three-valued logic $\mathbf{K}_{\mathbf{3}}^{\mathbf{2}}$ (LP) [16] and its extensions ${ }^{2}$.

In [20] A. Tamminga explains the idea of correspondence analysis applied to $\mathbf{L P}$ as follows:

" $<\ldots>$ characterize every possible single entry in the truth table of a unary or a binary truth-functional operator by a basic inference scheme. As a consequence, each unary and each binary truth-functional operator is characterized by a set of basic inference schemes. Kooi and Tamminga show that if we add the inference schemes that characterize an operator to a natural deduction system for $\mathbf{L P}$, we immediately obtain a natural deduction system that is sound and complete with respect to the logic that contains, next to LP's negation, disjunction, and conjunction, the additional operator" [20, p. 256].

Thus, this paper continues B. Kooi and A. Tamminga's proof-theoretic studies of three-valued logics, spreading them on the field of four-valued logics and thereby offering universal instrument of axiomatization of all possible truth-table extensions of $\mathbf{F D E}+$.

\section{Semantics}

N.D. Belnap's semantics [3, 4]. Consider a matrix $\mathfrak{M}_{4}=\langle\{1, b, n, 0\}$, $\neg, \wedge, \vee,\{1, b\}\rangle$ of the logic FDE, a matrix $\mathfrak{M}_{4}^{+}=\langle\{1, b, n, 0\}, \neg, \sim, \wedge, \vee$, $\{1, b\rangle\}$ of the logic $\mathbf{F D E}+$, and a matrix $\mathfrak{M}_{4}^{\#}=\langle\{1, b, n, 0\}, \neg, \sim, \wedge, \vee$, $\left.\star_{1}, \ldots, \star_{n}, \circ_{1}, \ldots, \circ_{m},\{1, b\rangle\right\}$ of the logic $\mathbf{F D E} \#$.

\footnotetext{
${ }^{1}$ Note that for some technical reasons before constructing such systems FDE (alphabet of which language contains $\neg$ (De Morgan negation), $\wedge$ (conjunction) and $\vee($ disjunction $)$ ) should be expanded by Boolean negation $\sim$. Let us denote this logic through FDE+.

${ }^{2}$ In A. Tamminga's paper [20] the similar result is obtained for $\mathbf{K}_{\mathbf{3}}[12,11]$ and its extensions.
} 


\begin{tabular}{|c|c|c|}
\hline$A$ & $\neg$ & $\sim$ \\
\hline 1 & 0 & 0 \\
\hline$b$ & $b$ & $n$ \\
\hline$n$ & $n$ & $b$ \\
\hline 0 & 1 & 1
\end{tabular}$\left|\begin{array}{|c|cccc}\wedge & 1 & b & n & 0 \\
\hline 1 & 1 & b & n & 0 \\
\hline b & b & b & 0 & 0 \\
\hline n & n & 0 & n & 0 \\
\hline 0 & 0 & 0 & 0 & 0\end{array}\right|$\begin{tabular}{|c|cccc|}
$\vee \vee$ & 1 & $b$ & $n$ & 0 \\
\hline 1 & 1 & 1 & 1 & 1 \\
\hline$b$ & 1 & $b$ & 1 & $b$ \\
\hline$n$ & 1 & 1 & $n$ & $n$ \\
\hline 0 & 1 & $b$ & $n$ & 0 \\
\hline
\end{tabular}

Unary operators $\star_{1}, \ldots, \star_{n}$ and binary operators $\circ_{1}, \ldots, \circ_{m}$ are arbitrary. In the particular case they can be connectives of FDE + or all possible fourvalued connectives. By these reason I do not give here truth tables for them.

The values are ordered as follows: $0 \preccurlyeq n, 0 \preccurlyeq b, n \preccurlyeq 1, b \preccurlyeq 1 ; n$ and $b$ are incomparable (see a picture below).

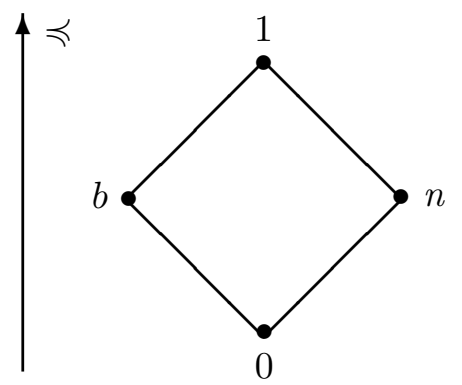

It is natural to regard the value 1 as "true", $b$ as "true and false", $n$ as "not true and not false" and 0 as "false". Note that N.D. Belnap himself defined the entailment through the relation $\preccurlyeq$. It was J.M. Font [9] who first proved that it is possible to redefine the entailment relation through designated values. The same result was independently obtained by D.V. Zaitsev and Y.V. Shramko [22]. Moreover, Y. Shramko and $\mathrm{H}$. Wansing [18] proved that it is possible to define entailment through set $\{0, b\}$ of antidesignated values.

J.M. Dunn's semantics [8]. Truth values here are subsets of a set of classical truth values $\{\mathfrak{t}, \mathfrak{f}\}$, that is $\{\mathfrak{t}\},\{\mathfrak{t}, \mathfrak{f}\}, \emptyset$ and $\{\mathfrak{f}\}$ which are analogues of values $1, b, n$ and 0 from Belnap's semantics. The conditions of truth and falsity for formulas are as follows ( $v$ is a valuation):

$$
\begin{gathered}
\mathfrak{t} \in v(\neg A) \Leftrightarrow \mathfrak{f} \in v(A) ; \\
\mathfrak{f} \in v(\neg A) \Leftrightarrow \mathfrak{t} \in v(A) ; \\
\mathfrak{t} \in v(\sim A) \Leftrightarrow \mathfrak{t} \notin v(A) ; \\
\mathfrak{f} \in v(\sim A) \Leftrightarrow \mathfrak{f} \notin v(A) ; \\
\mathfrak{t} \in v(A \wedge B) \Leftrightarrow \mathfrak{t} \in v(A) \dot{\wedge} \mathfrak{t} \in v(B) ; \\
\mathfrak{f} \in v(A \wedge B) \Leftrightarrow \mathfrak{f} \in v(A) \dot{\vee} \mathfrak{f} \in v(B) ; \\
\mathfrak{t} \in v(A \vee B) \Leftrightarrow \mathfrak{t} \in v(A) \dot{\vee} \mathfrak{t} \in v(B) ; \\
\mathfrak{f} \in v(A \vee B) \Leftrightarrow \mathfrak{f} \in v(A) \dot{\wedge} \mathfrak{f} \in v(B) .
\end{gathered}
$$


In terms of J.M. Dunn's semantics the relation of entailment in logics FDE, FDE + and FDE \# is defined as follows:

$$
\Gamma \models A \Leftrightarrow \dot{\forall} v(\underset{B \in \Gamma}{\dot{\forall} B} \mathfrak{t} \in v(B) \Rightarrow \mathfrak{t} \in v(A)) .
$$

\section{Inference schemes for arbitrary connectives}

Remark 1 (About designations). Let us denote through $\mathcal{L}^{\#}$ a language of FDE\#, through Prop a set of all propositional variables of the language $\mathcal{L}^{\#}$, through Form ${ }^{\#}$ a set of all $\mathcal{L}^{\#}$-formulas (formulas in the language $\left.\mathcal{L}^{\#}\right)$; through $f_{\star}$ a truth table for $\star$ and through $f_{\circ}$ a truth table for $\circ$. Let $x, y, z \in\{1, b, n, 0\}$, then let us denote through $f_{\star}(x)=y$ such entry (line) of a truth table $f_{\star}$ that $\dot{\forall} A \dot{\forall} v(v(A)=x \Rightarrow v(\star A)=y$ ); and through $f_{\circ}(x, y)=z$ such entry of a truth table $f_{\circ}$ that $\dot{\forall} A \dot{\forall} v((v(A)=x \dot{\wedge} v(B)=$ $y) \Rightarrow v(A \circ B)=z)$.

So, in this section propositions 1 and 2 are formulated. The first one states that for every entry of the form $f_{\star}(x)=y$ a characteristic inference scheme corresponds. The second one states that for every entry of the form $f_{\circ}(x, y)=z$ a characteristic inference scheme corresponds. It is clear that every operator $\star$ has 4 entries and it is characterised by 4 inference schemes; and every operator $\circ$ has 16 entries and it is characterised by 16 inference schemes. In the section 5 it is proved that by adding all inference schemes which characterise operators $\star_{1}, \ldots, \star_{n}, \circ_{1}, \ldots, \circ_{m}$ as rules of inference to FDE + we get not only sound, but complete natural deduction system for $\mathbf{F D E} \#$ (i.e. for $\mathbf{F D E}+$ extended by $\star_{1}, \ldots, \star_{n}, \circ_{1}, \ldots, \circ_{m}$ ).

Proposition 1. For every $\mathcal{L}^{\#}$-formula $A$ :

$$
\begin{aligned}
& f_{\star}(0)=\left\{\begin{array}{lll}
0 & \Leftrightarrow & \sim A, \neg A=\sim \star A \wedge \neg \star A \\
n & \Leftrightarrow & \sim A, \neg A \models \sim \star A \wedge \sim \neg \star A \\
b & \Leftrightarrow & \sim A, \neg A=\star A \wedge \neg \star A \\
1 & \Leftrightarrow & \sim A, \neg A \models \star A \wedge \sim \neg \star A
\end{array}\right. \\
& f_{\star}(n)=\left\{\begin{array}{lll}
0 & \Leftrightarrow & \sim A, \sim \neg A \models \sim \star A \wedge \neg \star A \\
n & \Leftrightarrow & \sim A, \sim \neg A \models \sim \star A \wedge \sim \neg \star A \\
b & \Leftrightarrow & \sim A, \sim \neg A \models \star A \wedge \neg \star A \\
1 & \Leftrightarrow & \sim A, \sim \neg A \models \star A \wedge \sim \neg \star A
\end{array}\right. \\
& f_{\star}(b)=\left\{\begin{array}{lll}
0 & \Leftrightarrow & A, \neg A \models \sim \star A \wedge \neg \star A \\
n & \Leftrightarrow & A, \neg A \models \sim \star A \wedge \sim \neg \star A \\
b & \Leftrightarrow & A, \neg A \models \star A \wedge \neg \star A \\
1 & \Leftrightarrow & A, \neg A \models \star A \wedge \sim \neg \star A
\end{array}\right.
\end{aligned}
$$


$f_{\star}(1)=\left\{\begin{array}{cc}0 & \Leftrightarrow A, \sim \neg A=\sim \star A \wedge \neg \star A \\ n & \Leftrightarrow \quad A, \sim \neg A=\sim \star A \wedge \sim \neg \star A \\ b & \Leftrightarrow \quad A, \sim \neg A \mid=\star A \wedge \neg \star A \\ 1 & \Leftrightarrow \quad A, \sim \neg A=\star A \wedge \sim \neg \star A\end{array}\right.$

Proof. Suppose $f_{\star}(0)=1$. Let us show that $\dot{\forall} A: \sim A, \neg A=\star A \wedge \sim \neg \star A$. According to the remark $1, f_{\star}(0)=1$ means that $f_{\star}$ has an entry such that $\dot{\forall} A \dot{\forall} v(v(A)=0 \Rightarrow v(\star A)=1)$. In the terms of J.M. Dunn's semantics the last statement is interpreted as $(\alpha) \dot{\forall} A \dot{\forall} v((\mathfrak{t} \notin v(A) \dot{\wedge} \mathfrak{f} \in v(A)) \Rightarrow(\mathfrak{t} \in$ $v(\star A) \dot{\wedge} \mathfrak{f} \notin v(\star A)))$. Now suppose $(\beta) \mathfrak{t} \in v(\sim A)$ and $\mathfrak{t} \in v(\neg A)$. Therefore, $(\gamma) \mathfrak{t} \notin v(A)$ and $\mathfrak{f} \in v(A)$. From $(\alpha)$ and $(\gamma)$ obtain that $(\delta) \mathfrak{t} \in v(\star A) \dot{\wedge}$ $\mathfrak{f} \notin v(\star A))$. Hence, $(\varepsilon) \mathfrak{t} \in v(\star A \wedge \sim \neg \star A)$. From $(\beta)$ and $(\varepsilon)$ obtain $(\zeta)$ $\dot{\forall} A \dot{\forall} v((\mathfrak{t} \in v(\sim A) \dot{\wedge} \mathfrak{t} \in v(\neg A)) \Rightarrow \mathfrak{t} \in v(\star A \wedge \sim \neg \star A))$. Therefore, $(\eta) \dot{\forall} A$ : $\sim A, \neg A=\star A \wedge \sim \neg \star A$.

Suppose $(\theta) \dot{\forall} A: \sim A, \neg A \models \star A \wedge \sim \neg \star A$, let us prove that $f_{\star}(0)=$ 1. From $(\theta)$ obtain $(\iota) \dot{\forall} A \dot{\forall} v((\mathfrak{t} \in v(\sim A) \dot{\wedge} \mathfrak{t} \in v(\neg A)) \Rightarrow \mathfrak{t} \in v(\star A \wedge$ $\sim \neg \star A))$. From $(\iota)$ follows $(\kappa) \dot{\forall} A \dot{\forall} v((\mathfrak{t} \notin v(A) \dot{\wedge} \mathfrak{f} \in v(A)) \Rightarrow(\mathfrak{t} \in v(\star A)$ $\dot{\wedge} \mathfrak{f} \notin v(\star A)))$, which is equivalent to $(\lambda) \dot{\forall} A \dot{\forall} v(v(A)=0 \Rightarrow v(\star A)=1)$. According to the remark $1, f_{\star}(0)=1$ is an abbreviation for $(\lambda)$.

The other cases are proved similarly.

Now let us formulate the analogues proposition for binary operators.

Proposition 2. For every $\mathcal{L}^{\#}$-formulas $A$ and $B$ :

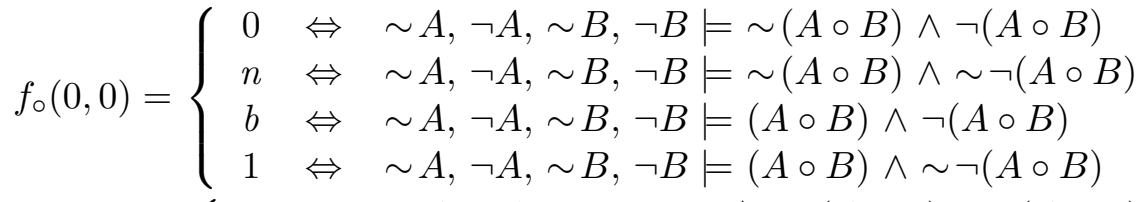

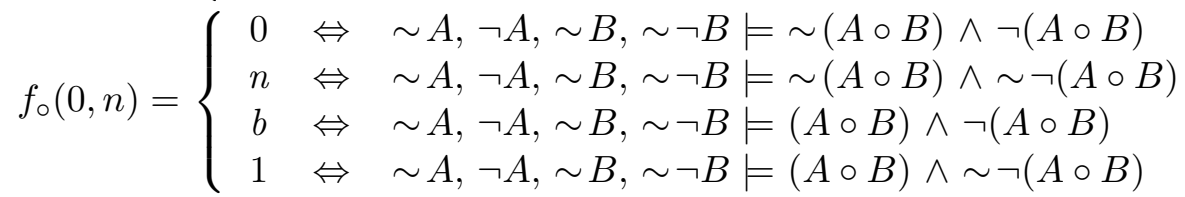

$$
\begin{aligned}
& f_{\circ}(0, b)=\left\{\begin{aligned}
0 & \Leftrightarrow \sim A, \neg A, B, \neg B \models \sim(A \circ B) \wedge \neg(A \circ B) \\
n & \Leftrightarrow \sim A, \neg A, B, \neg B \models \sim(A \circ B) \wedge \sim \neg(A \circ B) \\
b & \Leftrightarrow \sim A, \neg A, B, \neg B \models(A \circ B) \wedge \neg(A \circ B) \\
1 & \Leftrightarrow \sim A, \neg A, B, \neg B \models(A \circ B) \wedge \sim \neg(A \circ B)
\end{aligned}\right. \\
& f_{\circ}(0,1)=\left\{\begin{aligned}
0 & \Leftrightarrow \sim A, \neg A, B, \sim \neg B \mid=\sim(A \circ B) \wedge \neg(A \circ B) \\
n & \Leftrightarrow \sim A, \neg A, B, \sim \neg B \mid=\sim(A \circ B) \wedge \sim \neg(A \circ B) \\
b & \Leftrightarrow \sim A, \neg A, B, \sim \neg B \mid=(A \circ B) \wedge \neg(A \circ B) \\
1 & \Leftrightarrow \sim A, \neg A, B, \sim \neg B \mid=(A \circ B) \wedge \sim \neg(A \circ B)
\end{aligned}\right.
\end{aligned}
$$




$$
\begin{aligned}
& f_{\circ}(n, 0)=\left\{\begin{array}{l}
0 \Leftrightarrow \\
n \quad \Leftrightarrow \quad \sim A, \sim \neg A, \sim B, \neg B=\sim(A \circ B) \wedge \neg(A \circ B) \\
b \quad \Leftrightarrow \quad \sim A, \sim \neg A, \sim B, \neg B=(A \circ B) \wedge \neg(A \circ B) \\
1 \quad \Leftrightarrow \quad \sim A, \sim \neg A, \sim B, \neg B=(A \circ B) \wedge \sim \neg(A \circ B)
\end{array}\right.
\end{aligned}
$$

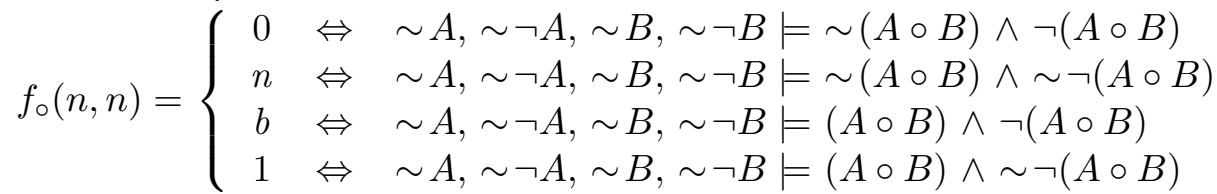

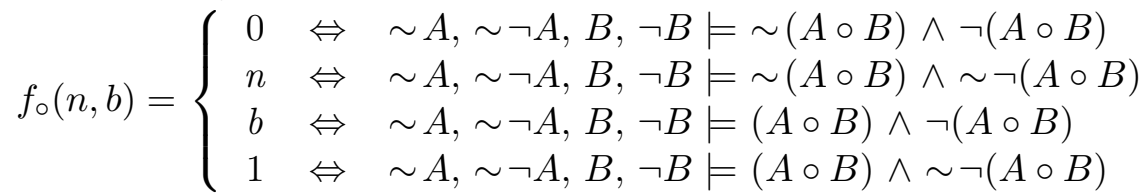

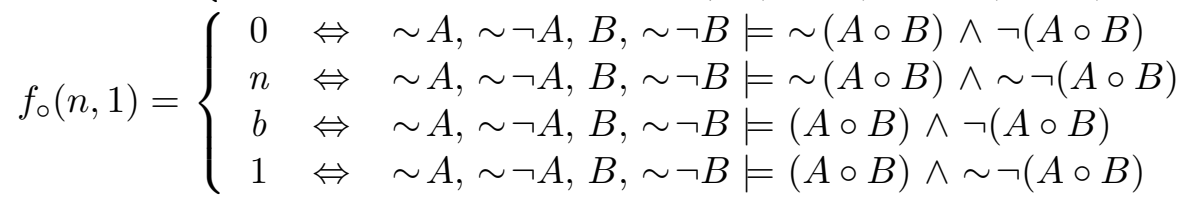

$$
\begin{aligned}
& f_{\circ}(b, 0)= \begin{cases}0 & \Leftrightarrow \quad A, \neg A, \sim B, \neg B \models \sim(A \circ B) \wedge \neg(A \circ B) \\
n & \Leftrightarrow \quad A, \neg A, \sim B, \neg B=\sim(A \circ B) \wedge \sim \neg(A \circ B) \\
b & \Leftrightarrow \quad A, \neg A, \sim B, \neg B=(A \circ B) \wedge \neg(A \circ B) \\
1 & \Leftrightarrow \quad A, \neg A, \sim B, \neg B=(A \circ B) \wedge \sim \neg(A \circ B)\end{cases} \\
& f_{\circ}(b, n)=\left\{\begin{array}{l}
0 \Leftrightarrow A, \neg A, \sim B, \sim \neg B \models \sim(A \circ B) \wedge \neg(A \circ B) \\
n \quad \Leftrightarrow \quad A, \neg A, \sim B, \sim \neg B \models \sim(A \circ B) \wedge \sim \neg(A \circ B) \\
b \quad \Leftrightarrow \quad A, \neg A, \sim B, \sim \neg B \models(A \circ B) \wedge \neg(A \circ B) \\
1 \quad \Leftrightarrow \quad A, \neg A, \sim B, \sim \neg B \models(A \circ B) \wedge \sim \neg(A \circ B)
\end{array}\right. \\
& f_{\circ}(b, b)=\left\{\begin{aligned}
0 & \Leftrightarrow A, \neg A, B, \neg B \models \sim(A \circ B) \wedge \neg(A \circ B) \\
n & \Leftrightarrow A, \neg A, B, \neg B \models \sim(A \circ B) \wedge \sim \neg(A \circ B) \\
b & \Leftrightarrow A, \neg A, B, \neg B \models(A \circ B) \wedge \neg(A \circ B) \\
1 & \Leftrightarrow A, \neg A, B, \neg B \models(A \circ B) \wedge \sim \neg(A \circ B)
\end{aligned}\right. \\
& f_{\circ}(b, 1)=\left\{\begin{array}{l}
0 \Leftrightarrow A, \neg A, B, \sim \neg B=\sim(A \circ B) \wedge \neg(A \circ B) \\
n \quad \Leftrightarrow \quad A, \neg A, B, \sim \neg B=\sim(A \circ B) \wedge \sim \neg(A \circ B) \\
b \quad \Leftrightarrow \quad A, \neg A, B, \sim \neg B=(A \circ B) \wedge \neg(A \circ B) \\
1 \quad \Leftrightarrow \quad A, \neg A, B, \sim \neg B=(A \circ B) \wedge \sim \neg(A \circ B)
\end{array}\right. \\
& f_{\circ}(1,0)=\left\{\begin{array}{l}
0 \quad \Leftrightarrow \quad A, \sim \neg A, \sim B, \neg B \mid=\sim(A \circ B) \wedge \neg(A \circ B) \\
n \quad \Leftrightarrow \quad A, \sim \neg A, \sim B, \neg B=\sim(A \circ B) \wedge \sim \neg(A \circ B) \\
b \quad \Leftrightarrow \quad A, \sim \neg A, \sim B, \neg B \mid=(A \circ B) \wedge \neg(A \circ B) \\
1 \quad \Leftrightarrow \quad A, \sim \neg A, \sim B, \neg B \mid=(A \circ B) \wedge \sim \neg(A \circ B)
\end{array}\right.
\end{aligned}
$$




$$
\begin{aligned}
& f_{\circ}(1, n)=\left\{\begin{array}{lll}
0 & \Leftrightarrow A, \sim \neg A, \sim B, \sim \neg B=\sim(A \circ B) \wedge \neg(A \circ B) \\
n & \Leftrightarrow \quad A, \sim \neg A, \sim B, \sim \neg B=\sim(A \circ B) \wedge \sim \neg(A \circ B) \\
b & \Leftrightarrow \quad A, \sim \neg A, \sim B, \sim \neg B=(A \circ B) \wedge \neg(A \circ B) \\
1 & \Leftrightarrow \quad A, \sim \neg A, \sim B, \sim \neg B=(A \circ B) \wedge \sim \neg(A \circ B)
\end{array}\right. \\
& f_{\circ}(1, b)=\left\{\begin{array}{lll}
0 & \Leftrightarrow A, \sim \neg A, B, \neg B=\sim(A \circ B) \wedge \neg(A \circ B) \\
n & \Leftrightarrow \quad A, \sim \neg A, B, \neg B=\sim(A \circ B) \wedge \sim \neg(A \circ B) \\
b & \Leftrightarrow \quad A, \sim \neg A, B, \neg B=(A \circ B) \wedge \neg(A \circ B) \\
1 & \Leftrightarrow \quad A, \sim \neg A, B, \neg B=(A \circ B) \wedge \sim \neg(A \circ B)
\end{array}\right. \\
& f_{\circ}(1,1)=\left\{\begin{array}{lll}
0 & \Leftrightarrow A, \sim \neg A, B, \sim \neg B=\sim(A \circ B) \wedge \neg(A \circ B) \\
n & \Leftrightarrow \quad A, \sim \neg A, B, \sim \neg B=\sim(A \circ B) \wedge \sim \neg(A \circ B) \\
b & \Leftrightarrow \quad A, \sim \neg A, B, \sim \neg B=(A \circ B) \wedge \neg(A \circ B) \\
1 & \Leftrightarrow \quad A, \sim \neg A, B, \sim \neg B=(A \circ B) \wedge \sim \neg(A \circ B)
\end{array}\right.
\end{aligned}
$$

Proof. Suppose $f_{\circ}(b, n)=0$. Let us show that $\dot{\forall} A \dot{\forall} B: A, \neg A, \sim B, \sim \neg B$ $\models \sim(A \circ B) \wedge \neg(A \circ B)$. According to the remark $1, f_{\circ}(b, n)=0$ means that $f_{\circ}$ has an entry such that $\dot{\forall} A \dot{\forall} B \dot{\forall} v((v(A)=b \dot{\wedge} v(B)=n) \Rightarrow v(A \circ B)=0)$. In the terms of J.M. Dunn's semantics the last statement is understood as $(\alpha) \dot{\forall} A \dot{\forall} B \dot{\forall} v((\mathfrak{t} \in v(A) \dot{\wedge} \mathfrak{f} \in v(A) \dot{\wedge} \mathfrak{t} \notin v(B) \dot{\wedge} \mathfrak{f} \notin v(B)) \Rightarrow(\mathfrak{t} \notin v(A \circ B)$ $\dot{\wedge} \mathfrak{f} \in v(A \circ B)))$. Now suppose $(\beta) \mathfrak{t} \in v(A), \mathfrak{t} \in v(\neg A), \mathfrak{t} \in v(\sim B)$ and $\mathfrak{t} \in v(\sim \neg B)$. Therefore, $(\gamma) \mathfrak{t} \in v(A) \dot{\wedge} \mathfrak{f} \in v(A) \dot{\wedge} \mathfrak{t} \notin v(B) \dot{\wedge} \mathfrak{f} \notin v(B)$. From $(\alpha)$ and $(\gamma)$ obtain that $(\delta) \mathfrak{t} \notin v(A \circ B) \dot{\wedge} \mathfrak{f} \in v(A \circ B)$. Hence, $(\varepsilon)$ $\mathfrak{t} \in v(\sim(A \circ B) \wedge \neg(A \circ B))$. From $(\beta)$ and $(\varepsilon)$ obtain $(\zeta) \dot{\forall} A \dot{\forall} v((\mathfrak{t} \in v(A)$ $\dot{\wedge} \mathfrak{t} \in v(\neg A) \dot{\wedge} \mathfrak{t} \in v(\sim B) \dot{\wedge} \mathfrak{t} \in v(\sim \neg B)) \Rightarrow \mathfrak{t} \in v(\sim(A \circ B) \wedge \neg(A \circ B)))$. Therefore, $(\eta) \dot{\forall} A \dot{\forall} B: A, \neg A, \sim B, \sim \neg B \models \sim(A \circ B) \wedge \neg(A \circ B)$.

Suppose $(\theta) \dot{\forall} A \dot{\forall} B: A, \neg A, \sim B, \sim \neg B \models \sim(A \circ B) \wedge \neg(A \circ B)$, let us prove that $f_{\circ}(b, n)=0$. From $(\theta)$ obtain $(\iota) \dot{\forall} A \dot{\forall} v((\mathfrak{t} \in v(A) \dot{\wedge} \mathfrak{t} \in v(\neg A)$ $\dot{\wedge} \mathfrak{t} \in v(\sim B) \dot{\wedge} \mathfrak{t} \in v(\sim \neg B)) \Rightarrow \mathfrak{t} \in v(\sim(A \circ B) \wedge \neg(A \circ B)))$. From $(\iota)$ follows $(\kappa) \dot{\forall} A \dot{\forall} B \dot{\forall} v((\mathfrak{t} \in v(A) \dot{\wedge} \mathfrak{f} \in v(A) \dot{\wedge} \mathfrak{t} \notin v(B) \dot{\wedge} \mathfrak{f} \notin v(B)) \Rightarrow(\mathfrak{t} \notin$ $v(A \circ B) \dot{\wedge} \mathfrak{f} \in v(A \circ B)))$, which is equivalent to $(\lambda) \dot{\forall} A \dot{\forall} B \dot{\forall} v((v(A)=b \dot{\wedge}$ $v(B)=n) \Rightarrow v(A \circ B)=0)$. According to the remark $1, f_{\circ}(b, n)=0$ is an abbreviation for $(\lambda)$.

The other cases are proved similarly.

\section{Natural deduction system}

A natural deduction system for FDE is as follows ${ }^{3}$ :

$$
(\neg \neg I) \frac{A}{\neg \neg A} \quad(\neg \neg E) \frac{\neg \neg A}{A} \quad\left(\vee I_{1}\right) \frac{A}{A \vee B} \quad\left(\vee I_{2}\right) \frac{B}{A \vee B}
$$

\footnotetext{
${ }^{3}$ This system was first introduced by G. Priest [15].
} 


$$
\begin{array}{ccc}
(\vee E) \frac{A \vee B,}{A]^{+1}} & {\left[B, \quad[]^{+2}\right.} & (\wedge I) \frac{A, B}{A \wedge B} \\
C & \left(\wedge E_{1}\right) \frac{A \wedge B}{A} & \left(\wedge E_{2}\right) \frac{A \wedge B}{B} \quad(\neg \vee I) \frac{\neg A \wedge \neg B}{\neg(A \vee B)} \\
(\neg \vee E) \frac{\neg(A \vee B)}{\neg A \wedge \neg B} & (\neg \wedge I) \frac{\neg A \vee \neg B}{\neg(A \wedge B)} \quad(\neg \wedge E) \frac{\neg(A \wedge B)}{\neg A \vee \neg B}
\end{array}
$$

Rules for Boolean negation are as follows:

$$
(E F Q) \frac{A, \sim A}{B} \quad(E M) \frac{}{A \vee \sim A} \quad(\sim \neg E) \frac{\sim \neg A}{\neg \sim A} \quad(\neg \sim E) \frac{\neg \sim A}{\sim \neg A}
$$

A rule of inference of the form $\mathcal{R}_{\star}(x, y) \frac{A_{1}, \ldots, A_{n}}{B}$ corresponds to an entry $f_{\star}(x)=y$ of a truth table $f_{\star}$ and a rule of the form $\mathcal{R}_{\circ}(x, y, z)$ $\frac{A_{1}, \ldots, A_{m}}{B}$ corresponds to an entry $f_{\circ}(x, y)=z$ of a truth table $f_{\circ}$. Each connective $\star$ needs 4 rules of the form $\mathcal{R}_{\star}(x, y)$ and each connective $\circ$ needs 16 rules of the form $\mathcal{R}_{\circ}(x, y, z)$. These rules are inference schemes introduced in the section 3. Here is an example. According to the proposition 1, the rule $\mathcal{R}_{\star}(0,0)$ corresponds to the entry $f_{\star}(0)=0$ of the truth table $f_{\star}$ :

$$
\mathcal{R}_{\star}(0,0) \frac{\sim A, \neg A}{\sim \star A \wedge \neg \star A} .
$$

\section{Completeness theorem}

It is not difficult to prove the following theorem 1.

TheOREm 1 (Soundness). For every set of $\mathcal{L}_{-}^{\#}$-formulas $\Gamma$ and for every $\mathcal{L}^{\#}$-formula $A: \Gamma \vdash A \Rightarrow \Gamma \models A$.

While proving completeness theorem prime theories are used as syntactic analogues of valuations.

Definition 1. For every set of $\mathcal{L}^{\#}$-formulas $\Gamma$ and for every $\mathcal{L}^{\#}$-formulas $A$ and $B \Gamma$ is a prime theory, if the following conditions are true:

(Г1) $\Gamma \neq$ Form $^{\#}$ (non-triviality);

(Г2) $\Gamma \vdash A \Leftrightarrow A \in \Gamma$ (closure of $\vdash)$;

(Г3) $A \vee B \in \Gamma \Rightarrow(A \in \Gamma \dot{\vee} B \in \Gamma)$ (primeness). 
Elementhoods of $\mathcal{L}^{\#}$-formulas in prime theories are used as syntactic analogues of truth values.

Definition 2. For every prime theory $\Gamma$ and for every $\mathcal{L}^{\#}$-formula $A$ let us call $e(A, \Gamma)$ an elementhood of $A$ in $\Gamma$ and define it as follows:

$$
e(A, \Gamma)=\left\{\begin{array}{ccc}
1 & \Leftrightarrow & A \in \Gamma, \neg A \notin \Gamma ; \\
b & \Leftrightarrow & A \in \Gamma, \neg A \in \Gamma ; \\
n & \Leftrightarrow & A \notin \Gamma, \neg A \notin \Gamma ; \\
0 & \Leftrightarrow & A \notin \Gamma, \neg A \in \Gamma .
\end{array}\right.
$$

The following lemma 1 shows us that the definition 2 is consistent with the truth tables for the propositional connectives.

Lemma 1 . For every prime theory $\Gamma$ and for every $\mathcal{L}^{\#}$-formulas $A$ and $B$ :

(1) $f_{\neg}(e(A, \Gamma))=e(\neg A, \Gamma)$;

(2) $f_{\sim}(e(A, \Gamma))=e(\sim A, \Gamma)$

(3) $f_{\vee}(e(A, \Gamma), e(B, \Gamma))=e(A \vee B, \Gamma)$;

(4) $f_{\wedge}(e(A, \Gamma), e(B, \Gamma))=e(A \wedge B, \Gamma)$;

(5) $f_{\star}(e(A, \Gamma))=e(\star A, \Gamma)$;

(6) $f_{\circ}(e(A, \Gamma), e(B, \Gamma))=e(A \circ B, \Gamma)$.

ProOF.

(1) (A) $e(A, \Gamma)=0$. Then $A \notin \Gamma, \neg A \in \Gamma$. Suppose $\neg \neg A \in \Gamma$. According to $(\neg \neg E), A \in \Gamma$. Contradiction. Hence, $\neg \neg A \notin \Gamma$. Therefore, $e(\neg A, \Gamma)=1=f_{\neg}(0)=f_{\neg}(e(A, \Gamma))$.

(B) $e(A, \Gamma)=n$. Then $A \notin \Gamma, \neg A \notin \Gamma$. Similar to (A).

(C) $e(A, \Gamma)=b$. Then $A \in \Gamma, \neg A \in \Gamma$. According to $(\neg \neg I), \neg \neg A \in \Gamma$. Therefore, $e(\neg A, \Gamma)=n=f_{\neg}(n)=f_{\neg}(e(A, \Gamma))$.

(D) $e(A, \Gamma)=1$. Then $A \in \Gamma, \neg A \notin \Gamma$. Similar to (C).

(2) (A) $e(A, \Gamma)=0 . A \notin \Gamma, \neg A \in \Gamma$. According to (EM) and (Г3), $A \in \Gamma \dot{\vee} \sim A \in \Gamma$. Since $A \notin \Gamma, \sim A \in \Gamma$. Let $\neg \sim A \in \Gamma$. By the rule $(\neg \sim E) \sim \neg A \in \Gamma$, by the rule $(E F Q) B \in \Gamma$. Contradiction. $\neg \sim A \notin \Gamma$. Hence, $e(\sim A, \Gamma)=1=f_{\sim}(0)=f_{\sim}(e(A, \Gamma))$. 
(B) $e(A, \Gamma)=n . A \notin \Gamma, \neg A \notin \Gamma$. According to $(E M)$ and (Г3), $\neg A \in \Gamma \dot{\vee} \sim \neg A \in \Gamma$. Since $\neg A \notin \Gamma, \sim \neg A \in \Gamma$. By the rule $(\sim \neg E)$ $\neg \sim A \in \Gamma$. According to $(E M)$ and (Г3), $A \in \Gamma \dot{\vee} \sim A \in \Gamma$. Since $A \notin \Gamma, \sim A \in \Gamma$. Hence, $e(\sim A, \Gamma)=b=f_{\sim}(n)=f_{\sim}(e(A, \Gamma))$.

(C) $e(A, \Gamma)=b . A \in \Gamma, \neg A \in \Gamma$. Let $\sim A \in \Gamma$. Then by the rule $(E F Q) B \in \Gamma$, that is $\Gamma=$ Form $^{\#}$, that contradicts to $(\Gamma 1)$. $\sim A \notin \Gamma$. Let $\neg \sim A \in \Gamma$. By the rule $(\neg \sim E) \sim \neg A \in \Gamma$, by the rule $(E F Q) B \in \Gamma$. Contradiction. $\neg \sim A \notin \Gamma$. Hence, $e(\sim A, \Gamma)=n=f_{\sim}(b)=f_{\sim}(e(A, \Gamma))$.

(D) $e(A, \Gamma)=1 . A \in \Gamma, \neg A \notin \Gamma$. Let $\sim A \in \Gamma$. Then by the rule $(E F Q) B \in \Gamma$, that is $\Gamma=$ Form $^{\#}$, that contradicts to $(\Gamma 1)$. $\sim A \notin \Gamma$. According to $(E M)$ and $(\Gamma 3), \neg A \in \Gamma \dot{\vee} \sim \neg A \in \Gamma$. Since $\neg A \notin \Gamma, \sim \neg A \in \Gamma$. By the rule $(\sim \neg E) \neg \sim A \in \Gamma$. Hence, $e(\sim A, \Gamma)=0=f_{\sim}(1)=f_{\sim}(e(A, \Gamma))$.

(3) (A) $e(A, \Gamma)=0, e(B, \Gamma)=0 . A \notin \Gamma, \neg A \in \Gamma, B \notin \Gamma, \neg B \in \Gamma$. Suppose $A \vee B \in \Gamma$. According to (Г3), $A \in \Gamma \dot{\vee} B \in$ $\Gamma$. Contradiction. Then $A \vee B \notin \Gamma$. According to $(\wedge I)$ and $(\neg \vee I), \neg(A \vee B) \in \Gamma$. Hence, $e(A \vee B, \Gamma)=0=f_{\vee}(0,0)=$ $f_{\vee}(e(A, \Gamma), e(B, \Gamma))$.

(B) $e(A, \Gamma)=n, e(B, \Gamma)=b . A \notin \Gamma, \neg A \notin \Gamma, B \in \Gamma, \neg B \in \Gamma$. By the rule $\left(\vee I_{2}\right), A \vee B \in \Gamma$. Suppose $\neg(A \vee B) \in \Gamma$, then by the rule $(\neg \vee E), \neg A \wedge \neg B \in \Gamma$, but by the rule $\left(\wedge E_{1}\right)$, $\neg A \in \Gamma$. Contradiction. Hence, $\neg(A \vee B) \notin \Gamma$. Consequently, $e(A \vee B, \Gamma)=1=f_{\vee}(n, b)=f_{\vee}(e(A, \Gamma), e(B, \Gamma))$.

The other cases are proved similarly.

(4) (A) $e(A, \Gamma)=0, e(B, \Gamma)=n . A \notin \Gamma, \neg A \in \Gamma, B \notin \Gamma, \neg B \notin \Gamma$. Suppose $A \wedge B \in \Gamma$. Then by the rules $\left(\wedge E_{1}\right)$ and $\left(\wedge E_{2}\right), A \in \Gamma$ and $B \in \Gamma$. Contradiction. $A \wedge B \notin \Gamma$. By the rules $\left(\vee I_{1}\right)$ and $(\neg \wedge I), \neg(A \wedge B) \in \Gamma$. Hence, $e(A \wedge B, \Gamma)=0=f_{\wedge}(0, n)=$ $f_{\wedge}(e(A, \Gamma), e(B, \Gamma))$.

(B) $e(A, \Gamma)=1, e(B, \Gamma)=1 . A \in \Gamma, \neg A \notin \Gamma, B \in \Gamma, \neg B \notin \Gamma$. By the rule $(\wedge I), A \wedge B \in \Gamma$. Suppose $\neg(A \wedge B) \in \Gamma$. By the rule $(\neg \wedge E), \neg A \vee \neg B \in \Gamma$, but then, according to $(\Gamma 3), \neg A \in \Gamma$ $\dot{\vee} \neg B \in \Gamma$. Contradiction. Hence, $\neg(A \wedge B) \notin \Gamma$. Consequently, $e(A \wedge B, \Gamma)=1=f_{\wedge}(1,1)=f_{\wedge}(e(A, \Gamma), e(B, \Gamma))$.

The other cases are proved similarly. 
(5) (A) Let $e(A, \Gamma)=0$. Then $A \notin \Gamma, \neg A \in \Gamma$.

$(\alpha)$ Suppose $e(\star A, \Gamma)=0$. Then $f_{\star}(0)=0$ and $\mathcal{R}_{\star}(0,0)$ is a rule for $\star$ in FDE\#. According to $(E M)$ and $(\Gamma 3), A \in \Gamma \dot{V}$ $\sim A \in \Gamma$. Since $A \notin \Gamma, \sim A \in \Gamma$. Then by the rules $\mathcal{R}_{\star}(0,0)$, $\left(\wedge E_{1}\right)$ and $\left(\wedge E_{2}\right) \sim \star A \in \Gamma$ and $\neg \star A \in \Gamma$. Let $\star A \in \Gamma$. Then by the rule $(E F Q) B \in \Gamma$, that contradicts to $(\Gamma 1) . \star A \notin \Gamma$. Hence, $e(\star A, \Gamma)=0=f_{\star}(0)=f_{\star}(e(A, \Gamma))$.

$(\beta)$ Suppose $e(\star A, \Gamma)=n$. Then $f_{\star}(0)=n$ and $\mathcal{R}_{\star}(0, n)$ is a rule for $\star$ in FDE\#. Using $(E M)$ and $(\Gamma 3)$, obtain that $\sim A \in \Gamma$. By the rules $\mathcal{R}_{\star}(0, n),\left(\wedge E_{1}\right)$ and $\left(\wedge E_{2}\right) \sim \star A \in \Gamma$ and $\sim \neg \star A \in \Gamma$. Using $(E F Q)$, obtain that $\star A \notin \Gamma$ and $\neg \star A \notin \Gamma$. Hence, $e(\star A, \Gamma)=n=f_{\star}(0)=f_{\star}(e(A, \Gamma))$.

$(\gamma)$ Suppose $e(\star A, \Gamma)=b$. Then $f_{\star}(0)=b$ and $\mathcal{R}_{\star}(0, b)$ is a rule for $\star$ in $\mathbf{F D E} \#$. Clearly, that $\sim A \in \Gamma$. By the rules $\mathcal{R}_{\star}(0, b),\left(\wedge E_{1}\right)$ and $\left(\wedge E_{2}\right) \star A \in \Gamma$ and $\neg \star A \in \Gamma$. Hence, $e(\star A, \Gamma)=b=f_{\star}(0)=f_{\star}(e(A, \Gamma))$.

$(\delta)$ Suppose $e(\star A, \Gamma)=1$. Then $f_{\star}(0)=1$ and $\mathcal{R}_{\star}(0,1)$ is a rule for $\star$ in $\mathbf{F D E} \#$. Clearly, that $\sim A \in \Gamma$. By the rules $\mathcal{R}_{\star}(0,1)$, $\left(\wedge E_{1}\right)$ and $\left(\wedge E_{2}\right) \star A \in \Gamma$ and $\sim \neg \star A \in \Gamma$. By the rule $(E F Q)$ $B \in \Gamma, \neg \star A \notin \Gamma$. Hence, $e(\star A, \Gamma)=1=f_{\star}(0)=f_{\star}(e(A, \Gamma))$.

The other cases are proved similarly.

(6) Analogues to (5)

Lemma 2. For every prime theory $\Gamma$ and for every valuation $v_{\Gamma}$ such that $\underset{p \in \text { Prop }}{\dot{\forall p}}\left(v_{\Gamma}(p)=e(p, \Gamma)\right): \underset{A \in \text { Form }^{\#}}{\dot{\forall} A}\left(v_{\Gamma}(A)=e(A, \Gamma)\right)$.

Proof. By structural induction on $\mathcal{L}^{\#}$-formula $A$ using the lemma 1.

Lemma 3 (Lindenbaum). For every set of $\mathcal{L}^{\#}$-formulas $\Gamma$, for every $\mathcal{L}^{\#}$ formula $A$ : if $\Gamma \nvdash A$, then $\exists \Gamma^{*}: \Gamma^{*} \subseteq$ Form $^{\#}$ and (1) $\Gamma \subseteq \Gamma^{*},(2) \Gamma^{*} \forall A$ and (3) $\Gamma^{*}$ is a prime theory.

Proof. Let $B_{1}, B_{2}, \ldots$ be an enumeration of all $\mathcal{L}^{\#}$-formulas. Now define a sequence of sets of $\mathcal{L}^{\#}$-formulas $\Gamma_{1}, \Gamma_{2}, \ldots$. Let $\Gamma_{1}=\Gamma$ and $\Gamma_{n}$ somehow defined. Then let $\Gamma_{n+1}=\Gamma_{n} \bigcup\left\{B_{n+1}\right\}$, if $\Gamma_{n} \bigcup\left\{B_{n+1}\right\} \not \forall A$; and $\Gamma_{n+1}=\Gamma_{n}$ otherwise. Let $\Gamma^{*}$ is the union of all $\Gamma_{i}$. 
(1) Follows from the definition of $\Gamma^{*}$.

(2) I will use the straightforward induction on $i$. Since $\Gamma_{1}=\Gamma, \Gamma_{1} \not \forall A$. By the inductive assumption, $\Gamma_{i} \not \forall A$. If $\Gamma_{i+1}=\Gamma_{i}$, then $\Gamma_{i+1} \not$ $A$. If $\Gamma_{i+1} \neq \Gamma_{i}$, then $\Gamma_{i+1}=\Gamma_{i} \bigcup\left\{B_{i+1}\right\}$. Suppose $\Gamma_{i} \bigcup\left\{B_{i+1}\right\} \vdash$ $A$. But then (by the definition of the sequence of $\left.\Gamma_{1}, \Gamma_{2}, \ldots\right) \Gamma_{i+1}=$ $\Gamma_{i}$. Contradiction. Hence, $\Gamma_{i} \bigcup\left\{B_{i+1}\right\} \forall A$. Thus, if $\Gamma_{i+1} \neq \Gamma_{i}$, then $\Gamma_{i+1} \not \forall A$. Clearly, that if for all $\Gamma_{i}$ true, that $\Gamma_{i} \forall A$, then $\Gamma^{*} \forall A$.

(3) Let us prove that: (A) $\Gamma^{*} \neq$ Form $^{\#}$ (non-triviality); (B) $\Gamma^{*} \vdash B \Leftrightarrow$ $B \in \Gamma^{*}$ (closure of $\vdash$ ); (C) $B \vee C \in \Gamma^{*} \Rightarrow\left(B \in \Gamma^{*} \dot{\vee} C \in \Gamma^{*}\right)$ (primeness).

(A) Since $\Gamma^{*} \forall A$, obviously, that $\Gamma^{*} \neq$ Form $^{\#}$.

(B) $(\Rightarrow)$. Suppose $\Gamma^{*} \vdash B$. Then $\dot{\exists} i$ : $B=B_{i}$ and $\dot{\exists} \Gamma_{i}$ : $\Gamma_{i} \vdash B_{i}$. Suppose $B_{i} \notin \Gamma_{i}$. Hence, $\Gamma_{i-1} \bigcup\left\{B_{i}\right\} \vdash A$. But then $\Gamma^{*} \vdash A$, because $\Gamma_{i-1} \subseteq \Gamma^{*}$ and $\Gamma^{*} \vdash B$. Nonetheless, it was proved in (2) that $\Gamma^{*} \forall A$. Then $B_{i} \in \Gamma_{i}$. Thus, $\Gamma^{*} \vdash B \Rightarrow B \in \Gamma^{*}$.

$(\Leftarrow)$. Suppose $B \in \Gamma^{*}, \Gamma^{*} \forall B$. Then $\dot{\exists} i$ : $B=B_{i}$ and $\dot{\exists} \Gamma_{i-1}$ : $\Gamma_{i-1} \bigcup\left\{B_{i}\right\} \vdash A$. Since $\Gamma_{i-1} \subseteq \Gamma^{*}, \Gamma^{*} \bigcup\left\{B_{i}\right\} \vdash A$. From here and the fact, that $\Gamma^{*} \forall A$, obtain, that $B_{i} \notin \Gamma^{*}$, that is $B \notin \Gamma^{*}$. Contradiction. Hence, $\Gamma^{*} \vdash A$. Thus, $B \in \Gamma^{*} \Rightarrow \Gamma^{*} \vdash A$.

(C) Suppose $B \vee C \in \Gamma^{*}$, but $B \notin \Gamma^{*}, C \notin \Gamma^{*}$. Since $B \vee C \in \Gamma^{*}, \Gamma^{*} \vdash$ $B \vee C$ (see (B)). On the other hand, $\dot{\exists} i: B=B_{i}$ and $\dot{\exists} j: C=B_{j}$; $\Gamma_{i-1} \bigcup\left\{B_{i}\right\} \vdash A$ and $\Gamma_{j-1} \bigcup\left\{B_{j}\right\} \vdash A$. Moreover, $\Gamma_{i-1} \subseteq \Gamma^{*}$ and $\Gamma_{j-1} \subseteq \Gamma^{*}$. Then $\Gamma^{*} \bigcup\left\{B_{i}\right\} \vdash A$ and $\Gamma^{*} \bigcup\left\{B_{j}\right\} \vdash A$. From here and the fact, that $\Gamma^{*} \vdash B_{i} \vee B_{j}$, by the rule $(\vee E)$ obtain, that $\Gamma^{*} \vdash A$, but according to (2), $\Gamma^{*} \forall A$. Hence, $B \vee C \in \Gamma^{*} \Rightarrow$ $\left(B \in \Gamma^{*} \dot{\vee} C \in \Gamma^{*}\right)$.

Theorem 2 (COMpleteness). For every set of $\mathcal{L}^{\#}$-formulas $\Gamma$ and for every $\mathcal{L}^{\#}$-formula $A$ : $\Gamma \models A \Rightarrow \Gamma \vdash A$.

Proof. By contraposition. Let $\Gamma \nvdash A$. Then, by lemma $3, \dot{\exists} \Gamma^{*}\left(\Gamma \subseteq \Gamma^{*}\right.$, $\Gamma^{*} \forall A$ and $\Gamma^{*}$ is a prime theory). According to lemma 2 , there is a valuation $v_{\Gamma^{*}}$ such, that $\underset{B \in \Gamma}{\dot{\forall} B} v_{\Gamma^{*}}(B) \in\{1, b\} \dot{\wedge} v_{\Gamma^{*}}(A) \notin\{1, b\}$. But then $\Gamma \not \models A$.

TheOrem 3 (ADEQUACY). For every set of $\mathcal{L}^{\#}{ }_{-}$formulas $\Gamma$ and for every $\mathcal{L}^{\#}$-formula $A: \Gamma \models A \Leftrightarrow \Gamma \vdash A$.

Proof. The theorem follows from the theorems 1 and 2 . 


\section{Natural deduction for implicative extensions of FDE}

\subsection{History and semantics}

Using the technique of correspondence analysis, it is possible to axiomatize extensions of FDE, for example, implicative: $\mathbf{B N}_{\mathbf{4}}$, Par, FDEA, FDEB, FDEC, and FDED. Connective $\mapsto$ is implication of the logic $\mathbf{B N}_{\mathbf{4}}, \rightarrow_{e}$ is implication of the logic Par, $\rightarrow_{a}$ is implication of the logic FDEA, $\rightarrow_{b}$ is implication of the logic FDEB, $\rightarrow_{c}$ is implication of the logic FDEC, and $\rightarrow_{d}$ is implication of the logic FDED.

\begin{tabular}{|c|cccc}
\hline$\mapsto$ & 1 & $b$ & $n$ & 0 \\
\hline 1 & 1 & 0 & $n$ & 0 \\
\hline$b$ & 1 & $b$ & $n$ & 0 \\
\hline$n$ & 1 & $n$ & 1 & $n$ \\
\hline 0 & 1 & 1 & 1 & 1
\end{tabular}$\left|\begin{array}{ccccc}\rightarrow_{e} & 1 & b & n & 0 \\
1 & 1 & b & n & 0 \\
\hline b & 1 & b & n & 0 \\
n & 1 & 1 & 1 & 1 \\
0 & 1 & 1 & 1 & 1\end{array}\right|$\begin{tabular}{|c|cccc|}
$\rightarrow_{a}$ & 1 & $b$ & $n$ & 0 \\
\hline 1 & 1 & $b$ & $n$ & 0 \\
\hline$b$ & 1 & 1 & $n$ & $n$ \\
\hline$n$ & 1 & 1 & 1 & 1 \\
\hline 0 & 1 & 1 & 1 & 1 \\
\hline
\end{tabular}

$\left.\begin{array}{|c|cccc}\hline \rightarrow_{b} & 1 & b & n & 0 \\
\hline 1 & 1 & b & n & 0 \\
\hline b & 1 & 1 & n & n \\
\hline n & 1 & b & 1 & b \\
\hline 0 & 1 & 1 & 1 & 1\end{array} \mid \begin{array}{cccccc}\rightarrow_{c} & 1 & b & n & 0 \\
\hline 1 & 1 & b & n & 0 \\
\hline b & 1 & b & n & 0 \\
\hline n & 1 & b & 1 & b \\
0 & 1 & 1 & 1 & 1\end{array}\right]$\begin{tabular}{cccccc|}
$\rightarrow_{d}$ & 1 & $b$ & $n$ & 0 \\
\hline 1 & 1 & $b$ & $n$ & 0 \\
\hline$b$ & 1 & $b$ & $n$ & 0 \\
\hline$n$ & 1 & $b$ & 1 & $b$ \\
\hline 0 & 1 & $b$ & 1 & $b$ \\
\hline
\end{tabular}

\begin{tabular}{|c|c|c|c|c|}
\hline$A$ & $\neg e$ & $\neg a$ & $\neg c$ & $\neg d$ \\
\hline 1 & 0 & 0 & 0 & 0 \\
\hline$b$ & 0 & $n$ & 0 & 0 \\
\hline 1 & 1 & $b$ & $b$ & $b$ \\
\hline 0 & 1 & 1 & 1 & $b$ \\
\hline
\end{tabular}

The logic $\mathbf{B N}_{\mathbf{4}}$ first appeared in R.T. Brady's paper [6], where several semantics for it and a Hilbert-style calculus are introduced. There is another reference of this logic (independent of [6]) in J.K. Slaney's paper [19].

The logic Par was first formulated by V.M. Popov [14] in the form of sequent and Hilbert-style calculuses. A similar Hilbert-style system independently appeared in A. Avron's paper [2] under the name HBe. Avron also introduced four-valued semantics for $\rightarrow_{e}$ [2]. Moreover, functional equivalence of $\rightarrow_{e}$ and $\mapsto$ was proven in [2]. Furthermore, the truth table for $\rightarrow_{e}$ is mentioned in A.P. Pynko's paper [17] in relation to [14], but independent of [2]. In addition, it is easy to see that $A \rightarrow_{e} B \equiv_{\operatorname{def}} \neg_{e} A \vee B^{4}$.

\footnotetext{
${ }^{4} \mathrm{M}$. De and H. Omori [7] investigated four-valued classical negations $\neg_{e}, \neg_{a}, \neg_{c}$ and $\neg_{d}$ (in the notation of [7] $\neg_{e}, \neg_{1}, \neg_{2}$ and $\neg_{5}$ ) in line with the study of the relationship
} 
Using negations $\neg_{a}, \neg_{c}$ and $\neg_{d}$, it's possible to define implications of logics FDEA, FDEC ${ }^{5}$ and FDED: $A \rightarrow_{a} B \equiv_{d e f} \neg_{a} A \vee B ; A \rightarrow_{c} B \equiv_{d e f}$ $\neg_{c} A \vee B ; A \rightarrow_{d} B \equiv_{d e f} \neg_{d} A \vee B$.

A semantics of the logic FDEB is first explored in D.V. Zaitsev's doctoral dissertation [21]. Notice that $A \rightarrow_{b} B \equiv_{\text {def }} \sim A \vee B$.

It is noteworthy that in the paper [7] by M. De and H. Omori a logic $\mathrm{BD}+$ with connectives $\neg, \sim, \vee, \wedge$ and $\rightarrow_{b}$ is analyzed.

It is easy to see that for all $i(i \in\{a, b, c, d, e\}) A, A \rightarrow_{i} B \models B$, $\models A \rightarrow_{i}\left(B \rightarrow_{i} A\right), \models\left(A \rightarrow_{i}\left(B \rightarrow_{i} C\right)\right) \rightarrow_{i}\left(\left(A \rightarrow_{i} B\right) \rightarrow_{i}\left(A \rightarrow_{i} C\right)\right)$, and $\models\left(\left(A \rightarrow_{i} B\right) \rightarrow_{i} A\right) \rightarrow_{i} A$. Thus, implications $\rightarrow_{a}, \rightarrow_{b}, \rightarrow_{c}, \rightarrow_{d}$ and $\rightarrow_{e}$ are classical.

\subsection{Rules of inference}

Using the proposition 2 and the theorem 3 , it is not difficult to find necessary rules of inference for $\rightarrow_{i}(i \in\{a, b, c, d, e\})$. Nonetheless, it makes sense to reduce the number of the rules. As a result, natural deduction systems will become more convenient for work in them. It is possible to prove that the rules for $\mapsto$ can be reformulated as follows ${ }^{6}$ :

$$
\begin{gathered}
\left(\mapsto I_{1}\right) \frac{\neg A, B}{A \mapsto B} \quad\left(\mapsto I_{2}\right) \frac{\neg A}{A \vee(A \mapsto B)} \quad\left(\mapsto I_{3}\right) \frac{B \vee \neg B \vee(A \mapsto B)}{A \vee \neg} \quad(M T) \frac{A \mapsto B, \neg B}{\neg A} \\
\left(\mapsto I_{4}\right) \frac{B, A \mapsto B}{\neg B \vee(A \mapsto B)} \quad(M P) \frac{A, B}{B} \quad(\neg \mapsto E) \frac{\neg(A \mapsto B)}{A \wedge \neg B} \\
(\neg \mapsto I) \frac{A, \neg B}{\neg(A \mapsto B)} \quad(M \mapsto)
\end{gathered}
$$

Logics Par, FDEA, FDEB, FDEC and FDED contain the following rules in common:

$$
\left(\rightarrow I_{1}\right) \frac{B}{A \rightarrow B} \quad\left(\rightarrow I_{2}\right) \frac{}{A \vee(A \rightarrow B)} \quad(M P) \frac{A, A \rightarrow B}{B}
$$

The axiomatization of Par contains also the following rules:

of classical negation and properties of paraconsistency and paracompleteness of logical systems.

${ }^{5}$ Logics FDEA and FDEC have two relatives: BD1 with the connectives $\neg, \neg_{a}, \rightarrow_{a}$, $\vee$ and $\wedge$; and BD2 with the connectives $\neg, \neg_{c}, \rightarrow_{c}, \vee$ and $\wedge$ [7].

${ }^{6}$ There are two ways of proving this statement: (1) by proving the deductive equivalence of modified rules and rules based on the proposition 2; or (2) by completeness proof for implications just as it was done for the other connectives in the section 5 . 


$$
\left(\neg \rightarrow_{e} I\right) \frac{A, \neg B}{\neg\left(A \rightarrow_{e} B\right)} \quad\left(\neg \rightarrow_{e} E\right) \frac{\neg\left(A \rightarrow_{e} B\right)}{A \wedge \neg B}
$$

The axiomatization of FDEA contains also the following rules:

$$
\begin{gathered}
\left(\neg \rightarrow_{a} I\right) \frac{A, \neg B}{\neg A \vee \neg\left(A \rightarrow_{a} B\right)} \quad\left(\neg \rightarrow_{a} E_{1}\right) \frac{\neg\left(A \rightarrow_{a} B\right)}{A \wedge \neg B} \\
\left(\neg \rightarrow_{a} E_{2}\right) \frac{\neg\left(A \rightarrow_{a} B\right), \neg A}{C}
\end{gathered}
$$

The axiomatization of FDEB contains also the following rules:

$$
\begin{gathered}
\left(\neg \rightarrow_{b} I\right) \frac{\neg B}{\neg A \vee \neg\left(A \rightarrow_{b} B\right)} \quad\left(\neg \rightarrow_{b} E_{1}\right) \frac{\neg\left(A \rightarrow_{b} B\right)}{\neg B} \\
\left(\neg \rightarrow_{b} E_{2}\right) \frac{\neg\left(A \rightarrow_{b} B\right), \neg A}{C}
\end{gathered}
$$

The axiomatization of FDEC contains also the following rules:

$$
\begin{aligned}
& \left(\neg \rightarrow_{c} I_{1}\right) \frac{A, \neg B}{\neg\left(A \rightarrow_{c} B\right)} \quad\left(\neg \rightarrow_{c} I_{2}\right) \frac{\neg B}{\neg A \vee \neg\left(A \rightarrow_{c} B\right)} \\
& \left(\neg \rightarrow_{c} E_{1}\right) \frac{\neg\left(A \rightarrow_{c} B\right)}{\neg B} \quad\left(\neg \rightarrow_{c} E_{2}\right) \frac{\neg\left(A \rightarrow_{c} B\right), \neg A}{A}
\end{aligned}
$$

The axiomatization of FDED contains also the following rules:

$$
\begin{gathered}
\left(\neg \rightarrow_{d} I_{1}\right) \frac{A, \neg B}{\neg\left(A \rightarrow_{d} B\right)} \quad\left(\neg \rightarrow_{d} I_{2}\right) \frac{\neg B}{\neg A \vee \neg\left(A \rightarrow_{d} B\right)} \\
\left(\neg \rightarrow_{d} I_{3}\right) \frac{\neg A, \neg B}{A \vee \neg\left(A \rightarrow_{d} B\right)} \quad\left(\neg \rightarrow_{d} E\right) \frac{\neg\left(A \rightarrow_{d} B\right)}{\neg B}
\end{gathered}
$$

\section{Conclusion}

In summary, the result obtained in this paper allows to get immediately adequate natural deduction systems for all possible truth-table expansions of FDE +. Consequently, a problem for future research arises: to formulate propositions 1 and 2 without the use of Boolean negation, in other words, to apply the technique of correspondence analysis to FDE directly, without recourse to $\mathbf{F D E}+$. In future prospect one more direction of research opens: to apply the technique of correspondence analysis to other four-valued logics or even to arbitrary $k$-valued logics with $l$ designated values, where $k \geqslant 3$ and $l \in\{1, \ldots, k-1\}$. 
Acknowledgements. I would like to express my sincere gratitude and appreciation to Dmitry Zaitsev for scientific guidance and assistance in the work on this paper, to Vladimir Shalack for fruitful discussions during the preparation of this paper, to Allard Tamminga for the deliberation of the previous version of this paper, and to the referee of this journal for advice on the improvement of this paper.

\section{References}

[1] Anderson, A.R., Belnap, N.D. "Tautological entailments", Philosophical Studies, 1962, vol. 13(1-2), pp. 9-24.

[2] Avron, A. "Natural 3-valued logics - characterization and proof theory", The Journal of Symbolic Logic, 1991, vol. 56(1), pp. 276-294.

[3] Belnap, N.D. "A useful four-valued logic", Modern Uses of Multiple-Valued Logic, ed. by J.M. Dunn, G. Epstein. Boston: Reidel Publishing Company, 1977, pp. 7-37.

[4] Belnap, N.D. "How a computer should think", Contemporary Aspects of Philosophy, ed. by G. Rule. Stocksfield: Oriel Press, 1977, pp. 30-56.

[5] Belnap, N.D. "Tautological entailments", The Journal of Symbolic Logic, 1959, vol. 24(4), p. 316.

[6] Brady, R.T. "Completeness proofs for the systems RM3 and BN4", Logique et Analyse, 1982, vol. 25(97), pp. 51-61.

[7] De, M., Omori, H. "Classical Negation and Expansions of Belnap-Dunn Logic", Studia Logica, 2015, vol. 103(4), pp. 825-851.

[8] Dunn, J.M. "Intuitive semantics for first-degree entailment and coupled trees", Philosophical Studies, 1976, vol. 29(3), pp. 149-168.

[9] Font, J.M. "Belnap's Four-Valued Logic and De Morgan Lattices", Logic Journal of the IGPL, 1997, vol. 5(3), pp. 1-29.

[10] Karpenko, A.S. Razvitie mnogoznachnoi logiki [The development of manyvalued logic]. Moscow: LKI Publ., 2010 (3d edition). 448 p. (In Russian)

[11] Kleene, S.C. Introduction to metamathematics. Amsterdam: WoltersNoordhoff Publishing and North-Holland Publishing Company, 1971 (6th reprint). $560 \mathrm{pp}$.

[12] Kleene, S.C. "On a notation for ordinal numbers", The Journal of Symbolic Logic, 1938, vol. 3(1), pp. 150-155.

[13] Kooi, B., Tamminga, A. "Completeness via correspondence for extensions of the logic of paradox", The Review of Symbolic Logic, 2012, vol. 5(4), pp. 720-730.

[14] Popov, V.M. "Sekventsial'nye formulirovki paraneprotivorechivykh logicheskikh sistem" [Sequent formulations of paraconsistent logical systems], Semanticheskie i sintaksicheskie issledovaniya neekstensional'nykh 
logik [Semantic and syntactic investigations of non-extensional logics], ed. by V.A. Smirnov. Moscow: Nauka Publ., 1989, pp. 285-289. (In Russian)

[15] Priest, G. "Paraconsistent logic", Handbook of philosophical logic. 2nd edition. Vol.6, ed. by M. Gabbay, F. Guenthner. Dordrecht: Kluwer, 2002, pp. 287393.

[16] Priest, G. "The logic of paradox", Journal of Philosophical Logic, 1979, vol. 8(1), pp. 219-241.

[17] Pynko, A.P. "Functional completeness and axiomatizability within Belnap's four-valued logic and and its expansion", Journal of Applied Non-Classical Logics, 1999, vol. 9(1), pp. 61-105.

[18] Shramko, Y., Wansing, H. "Entailment relations and/as truth values", Bulletin of the Section of Logic, 2007, vol. 36(3-4), pp. 131-143.

[19] Slaney, J.K. "The implications of paraconsistency", Proceedings of the 12th IJCAI, vol. 2, ed. by J. Mylopoulos and R. Reiter. Sydney: Morgan Kaufmann Publ., 1991, pp. 1052-1057.

[20] Tamminga, A. "Correspondence analysis for strong three-valued logic", Logical Investigations, 2014, vol. 20, pp. 255-268.

[21] Zaitsev, D.V. Obobshchennaya relevantnaya logika $i$ modeli rassuzhdenii [Generalized relevant logic and models of reasoning]. Moscow State Lomonosov University, doctoral (Doctor of Science) dissertation, 2012. 284 pp. (In Russian).

[22] Zaitsev, D.V., Shramko, Ya.V. "Logicheskoe sledovanie i vydelennye znacheniya" [Logical entailment and designated values], Logicheskie issledovaniya [Logical investigations], 2004, vol. 11, pp. 126-137. (In Russian) 